\title{
Risk Analysis of Vehicle Rear-End Collisions at Intersections
}

\author{
Sheng Dong $\mathbb{D}^{1}{ }^{1}$ Minjie Zhang, ${ }^{1}$ and Zhenjiang $\mathbf{L i}^{2}$ \\ ${ }^{1}$ School of Civil and Transportation Engineering, Ningbo University of Technology, Fenghua Rd. \#201, Jiangbei District, Ningbo, \\ Zhejiang Province, 315211, China \\ ${ }^{2}$ The State Key Laboratory for Management and Control of Complex Systems, Institute of Automation, \\ Chinese Academy of Sciences, Beijing 100190, China
}

Correspondence should be addressed to Sheng Dong; dongsheng@nbut.edu.cn

Received 3 January 2020; Revised 24 March 2020; Accepted 22 May 2020; Published 1 July 2020

Academic Editor: Feng Chen

Copyright (C) 2020 Sheng Dong et al. This is an open access article distributed under the Creative Commons Attribution License, which permits unrestricted use, distribution, and reproduction in any medium, provided the original work is properly cited.

\begin{abstract}
Aiming at solving a typical problem of past research using accident experience statistics of being unable to adapt to changing traffic flows, this paper provides an evaluation method of the risk of vehicle rear-end collisions at red-light-camera (RLC) intersections based on theoretical probabilities. Taking advantage of trajectory data of vehicles at the two similar intersections, which are Cao'an Road and Lvyuan Road with RLCs and Cao'an Road and Anhong Road without RLCs in Shanghai, a binary logit (BL) model of stop-and-go decision-making is established. Using the model and adjusting the headway and potential travel time, we can perform simulation and analysis of rear-end collisions. The result shows that this method is feasible to analyse the influence of RLCs on rear-end collisions. The analysis indicates that RLCs can cause higher speeds for vehicles passing the RLC intersection and more abnormal driving behaviors, which increase the difficulty of stop-and-go decision-making. RLCs do not always lead to an increase of rear-end collisions. For vehicles close to or far from intersection at the decision-making time, RLCs will significantly reduce the possibility of rear-end collisions; however, for vehicles in the potential travel time of $2 \mathrm{~s} \sim 3 \mathrm{~s}$, RLCs will increase the probability of rear-end collisions.
\end{abstract}

\section{Introduction}

In the past, running a red light during signal changes was a common phenomenon at intersections, which had a significant impact on the traffic safety of intersection. To effectively solve the problem of red-light violations, redlight cameras (RLCs) should be installed. RLCs have been widely used worldwide for more than 20 years. RLCs are also widely used in some large- and medium-sized cities in China. RLCs have played a huge role in reducing intentional red-light running. However, RLCs affect the driver's driving state and decision-making behavior at the intersection, and many potential safety problems that may be caused by RLCs have not been fully explored. Therefore, it is important to evaluate the impact of RLC installation on vehicle operation safety. Research on RLCs can not only find and eliminate the potential safety hazards presented by RLCs but also provide the basis for the future introduction of RLC installation national standards and use principles.
In China, the following problems and challenges still exist in the study of the safety impact of RLCs on vehicles at intersections:

(1) The limitations of RLC evaluation methods in mixed traffic: the environment and driving characteristics of mixed urban traffic are quite different from those of foreign countries. Red-light violations and accidents are affected by mixed traffic conditions. The evaluation of RLC effects in mixed traffic is more complex.

(2) Taking the accident rate as the metric of the evaluation method: the implementation of traffic accident collection systems is late, and there are many problems facing accident records. Simultaneously, the degree of urban renewal is quickly leading to frequent changes in facilities, which affects the occurrence of accidents, making the performance of RLC evaluation methods based on accident experience statistics in China questionable. 
This paper provides a method of evaluating the effect of RLCs on driver's traffic behavior based on mathematical simulation to solve the above problems. Using driving characteristic data of vehicles at the intersection decisionmaking time, the impact analysis and evaluation of RLCs on drivers' stop-and-go decision-making and rear-end hazards can be realized, therein avoiding the error and cost brought by accident statistics. This method can adjust the traffic parameters in the sensitivity analysis of the risk of rear-end accidents due to RLCs; thus, it can be applied to intersections with different traffic conditions and thus has portability. This method can be used to set the speed limit of the road after the installation of RLCs and can effectively reduce the potential safety hazards of the intersection after the installation of RLCs.

The organizational structure of this paper is as follows: first, the relevant background of this paper is introduced. Then, the literature review part summarizes the previous relevant research results and presents the focus of this study. The data collection methods used in this study appear in the "Field Observation and Data Collection" part, mainly including vehicles with and without RLCs, analysis of speed characteristics, decision-making behavior, and safety conditions. Based on the above data, the driving behavior model is constructed. First, the stop-and-go model is constructed and verified. Based on this, the rear-end collision risk model is constructed, and the rear-end collision probability is determined by changing the two key parameters of headway and potential travel time. The last part of this paper discusses the influence of RLCs on decision-making behavior during driving and in the risk of rear-end collisions.

\section{Literature Review}

There have been many research efforts on the application of RLCs, most of which focused on the occurrence of accidents related to RLC [1-6]. Chin's research shows that RLCs just applied in Singapore could effectively reduce red-light violations [7]. South, Hillier, Andreassen, Retting, and others, through accident comparisons before and after the installation of RLCs at intersections and the horizontal comparison of incidences of rear-end collisions, frontal collisions, and side collisions, analysed the impact of RLCs on traffic safety. The conclusions of these researchers are relatively consistent; that is, RLCs may increase rear-end collisions while reducing vehicle frontal collision and side collision accidents [8-15].

In addition, several studies have performed behavioral studies concerning RLCs. Helai Huang, Zeng. et al., through the analysis of the decision-making process, found that RLCs significantly affect the decision-making behavior of drivers. Although RLCs can reduce the red-light violation rates of vehicles, their effect on rear-end accidents at intersections is more complex. The possibility of RLCs increasing or reducing rear-end accidents is closely related to the speed of the following vehicle and the headway between the front vehicle and the following vehicle [16-20]. Lum et al. analysed two T-intersections and one crossintersection and found that the RLC can effectively increase drivers' stopping tendency; in addition, the RLC can accentuate the distance to the stop line to affect the stopping propensity. However, one drawback of this study was that the possible accident risk caused by RLCs was not included [21]. According to simulation analysis, Sun et al. found that RLCs can significantly reduce frontal accidents and may increase rear-end collisions. In addition, RLCs can affect a driver's comfort [22].

Generally speaking, past research on RLCs mainly has the following two problems: First, the safety impact analysis of RLCs is mostly based on accident experience statistics, where the accident data collection cycle is long and the time span is large, during which changes in other road traffic parameters will lead to reductions in the credibility of the final evaluation result. Thus, the RLC evaluation method based on accident experience statistics has serious defects. Second, the conclusion that RLCs can reduce the rightangle accidents and increase the rear-end accidents at intersections is unanimously accepted; however, the quantitative analysis of the rear-end collision risk by RLCs under limited conditions has not been carried out. Therefore, it is necessary to make a systematic analysis of the driver's behavior characteristics, the stop-and-go decision-making behavior, and the risk of traffic safety in the case of RLCs.

\section{Field Observation and Data Collection}

3.1. Site Descriptions. One of the best ways to analyse the influence of RLCs on drivers' driving behavior is to find two similar intersections: one is RLC intersection and the other is non-RLC intersection. The two intersections have similar characteristics in terms of geometric parameters, traffic flow composition and traffic volume, traffic management measures such as speed limit, and signal timing parameters such as phase number and the type of transition signals. It is very difficult to select the intersection meeting the above conditions. After a large-scale survey of intersections in Shanghai urban area, two adjacent signal intersections of Cao'an Road and Lvyuan Road and Cao'an road and Anhong Road are finally determined. The location of the two intersections is shown in Figure 1; the characteristics of intersections investigated are shown in Table 1.

3.2. Data Collection. In order to obtain all the traffic data related to driving behavior and ensure the accuracy of the data, video recording is used in this study. Take through lanes of the east import way as the research object. Two synchronous cameras need to be set, among which a far side camera was set $80 \mathrm{~m}$ upstream of the intersection, so as to capture the whole decision-making process of the vehicle in the approach. The other camera is used to record traffic signals.

Image processing software was used to process the video. The software can extract high-precision vehicle trajectory data, such as vehicle speed, acceleration, position coordinates, and traffic signal status at every time step. In order to meet the need of the study on stop-and-go decision-making 




FIgURE 1: Location of the observed intersections in Shanghai.

TABLE 1: Key parameters of the observed intersections.

\begin{tabular}{|c|c|c|c|}
\hline Description & & Lvyuan Road & Anhong Road \\
\hline \multirow{7}{*}{ Geometry parameters } & Intersection layout & "T" & “T” \\
\hline & Width of intersection & $45 \mathrm{~m}$ & $42 \mathrm{~m}$ \\
\hline & Adjacent bound level & Low & Low \\
\hline & Adjacent land use & University & Factory \\
\hline & Number of through lanes (westbound) & 5 & 4 (include 1 through-right lane) \\
\hline & Lane length & $3 \mathrm{~m}$ & $3 \mathrm{~m}$ \\
\hline & Pedestrian crossing & Yes & Yes \\
\hline \multirow{6}{*}{ Signal timings } & Cycle & $72 \mathrm{~s}$ & $90 \mathrm{~s}$ \\
\hline & No. of phases & $3 \mathrm{~s}$ & $3 \mathrm{~s}$ \\
\hline & Yellow time & $3 \mathrm{~s}$ & $3 s$ \\
\hline & Flash green & $3 \mathrm{~s}$ & $3 s$ \\
\hline & Transition signal & Green countdown & Green countdown \\
\hline & & (i) Flash green-amber & (i) Flash green-amber \\
\hline \multirow{2}{*}{ Traffic management } & RLC installation & Yes & No \\
\hline & Posted speed limit & $80 \mathrm{~km} / \mathrm{h}$ & $80 \mathrm{~km} / \mathrm{h}$ \\
\hline \multirow{2}{*}{ Traffic flow } & Flow composition & Percentage of car is $54 \%$ & Percentage of car is $59 \%$ \\
\hline & Traffic volume & $719 \mathrm{pcu} / \mathrm{h}$ & $748 \mathrm{pcu} / \mathrm{h}$ \\
\hline
\end{tabular}

and rear-end accidents and to avoid the interference of other vehicles in driving behavior, trajectory data of the last-tostop and the first-to-go through-ahead vehicles after the start of the green flashing light are extracted as the most important part. Data collection and video camera coverage are shown in Figure 2.

The method can also determine the time when the yellow light is on, the vehicle arrival information, the position of the vehicle in the fleet, the position of the vehicle when the yellow light is on, the decision-making behavior of the driver (stop, yellow light pass or violation), and so forth. The video processing is applied to 99 vehicles stopping and 251 vehicles passing with RLCs, among which 208 vehicles encounter yellow lights and 25 vehicles perform red-light violations, including 21 vehicles 3 seconds before the red lights start. A total of 20 vehicles stop under non-RLC conditions, 351 vehicles pass, 162 vehicles encounter yellow lights, and 157 vehicles violate red lights, including 85 vehicles 3 seconds before the red lights start.

\subsection{Data Reduction}

3.3.1. Statistical Analysis of Speed. Speed is an important indicator of driving behavior; thus, this study compares the speed of vehicles with and without the RLC from multiple perspectives. The time of vehicles arriving at the intersection can be divided into three categories: arrival during the tail of green time (i.e., less than $5 \mathrm{~s}$ from the end of green time, EG), arrival during the yellow-light time ( $3 \mathrm{~s}, \mathrm{AT})$, and arrival at the beginning of red time (i.e., the initial $3 \mathrm{~s}, \mathrm{BR}$ ). The statistical characteristics of the instantaneous speed of vehicles passing through the parking line in the above time intervals are shown in Figure 3. 


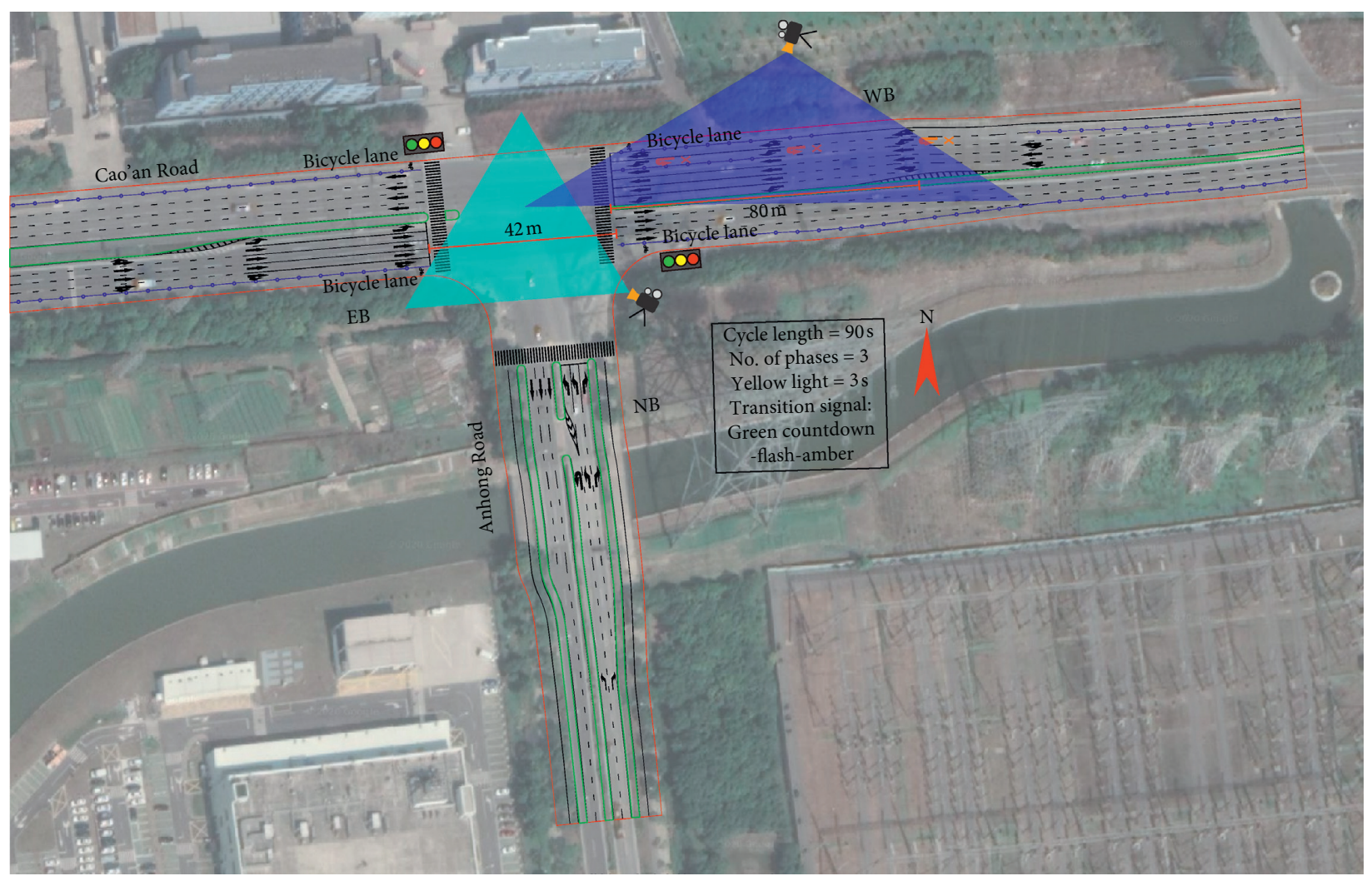

FIgure 2: Camera setting and data collection at the study intersections.



FIgURE 3: Statistical features of speed in different period. 
By comparing the passing speed of vehicles during EG, AT, and $\mathrm{BR}$, it is found that the speed of the vehicles with RLCs, such as the mean, minimum, maximum, and 15th percentile, 50th percentile, and 85 th percentile speeds, are relatively large compared with those of the vehicles without RLCs. Compared with the intersection without RLCs, the average speed with RLCs is lower when the yellow light is on; however, the mean, minimum, maximum, and 15th percentile, 50th percentile, and 85th percentile speeds all increase when the vehicle enters the intersection. From the speed analysis, RLCs have an important impact on the driver's speed. Compared to the case without RLCs, RLCs enable the driver to have a higher speed to leave the intersection entrance faster.

3.3.2. Abnormal Driving Behavior. According to previous studies [23], the driver makes a decision after the yellow light comes on (if the green flash or countdown is set before the yellow light, the decision-making time will be ahead of the yellow light) and estimates the distance from the current position to the stop line. Therefore, the distance from the stop line (actually reflected as the estimated time to the stop line) is the main judgment basis for deciding on passing or stopping. The driver's decision-making is mainly based on the estimated time to the stop line, while the estimated time difference refers to the difference between the actual running time from the decision-making time to the end of the yellow light and the estimated time to the stop line. The main reason for the abnormal decision-making is the estimated time difference. A negative difference means that the vehicle will enter the intersection at the current speed within the red time, so the driver should choose to stop; on the contrary, a positive difference means that the vehicle could safely pass the stop line at the current speed before the end of the yellow light, so the driver should do so. This is calculated as follows:

$$
\begin{aligned}
\mathrm{ETD} & =T_{a}-T_{e}, \\
T_{e} & =\frac{D_{d}}{V_{d}},
\end{aligned}
$$

where ETD is the estimated time difference, $T_{e}$ is the estimated time to stop line, that is, the potential travel time, $T_{a}$ is the actual duration from the decision point (i.e., start of amber/start of the flash green light) to the end of yellow light, $D_{d}$ is the distance from the position making decision point to the stop line, and $V_{d}$ is the instantaneous speed of the vehicle at the current decision point. For a random sample, the speed remains constant during the period from making decision to passing through the stop line.

If the result of the decision does not match the estimated time difference, it can be considered as an abnormal driving behavior, and its statistical results can reflect the change in the driver's state during the decision-making process. The difference in the driver's judgment of the distance results in the difference in driving behavior when passing or stopping. After making a decision, the driver will find that he cannot enter the intersection before the onset of red time at the current speed; thus, he will continue to accelerate. If the judgment error of the distance is large, the driver cannot pass. According to the matching results of the estimated time difference and the stop-and-go decision, the decisionmaking behavior can be divided into four types: normal pass, incorrect pass, normal stop, and abrupt stop. The decisionmaking mechanism is shown in Figure 4.

We analyse the stopping vehicles and passing vehicles during a yellow light and the initial stage of red time (3 s after the red light starts) and calculate the decision-making behavior data under RLC and non-RLC conditions, respectively, according to formulas (1) and (2), as shown in Table 2.

It can be seen from Table 3 that, in the case of non-RLC conditions, the proportion of incorrect pass during passing decisions and the proportion of abrupt stop during stopping decisions are significantly larger than those of the intersection with RLCs. It can be seen that installing RLCs can effectively reduce the occurrence of incorrect pass and abrupt stop.

\section{Driving Behavior Model Based on Data Simulation}

First, data were collected to establish an effective mathematical model, and then the analysis of various parameters was realized via mathematical simulation. The main advantage of mathematical simulation is its universality; that is, with the same set of computer equipment and different simulation software, various types of systems can be simulated and tested. This paper focuses on the impact of RLCs on rear-end collisions during decision-making through mathematical simulation. The main work of the simulation model is to build the stop-and-go decision-making model and rear-end collision probability model.

4.1. Stop-and-Go Decision-Making Model. The decisionmaking model of intersection driving behavior is the stopand-go model. A BL model (binary logit model, BL model for short) is easy to understand from a mathematical point of view and provides a simple method to explain the random probability generated by the cumulative logic density function assumed in the basic formula. Therefore, the BL model is the most widely used in addressing the decisionmaking of a driver when deciding to stop at or pass an intersection when encountering a yellow light. Through this model, the impact can be realized via a sensitivity analysis of factors related to the driver's decision-making behavior [9]. The BL model used to simulate driver stop-and-go decisionmaking is calculated as follows:

$$
\operatorname{Logit}\left(\pi_{i}\right)=\log \left(\frac{\pi_{i}}{1-\pi_{i}}\right)=\sum_{k=0}^{K} \beta_{k} x_{i k} .
$$

In the formula, $x_{i}$ is a group of vectors composed of $k$ interpretable variables, such as the geometry, traffic volume, and other intersection elements that can affect the driver's decision-making. $x_{i k}$ is the $k$-th variable, and $\beta_{k}$ is the corresponding influence coefficient of the $k$-th variable.

Considering the influence of the RLC, this paper analyses the driver's choice behavior near the intersection during the signal change interval, herein fully considering factors such as the vehicle speed, the distance to the stop line when the 


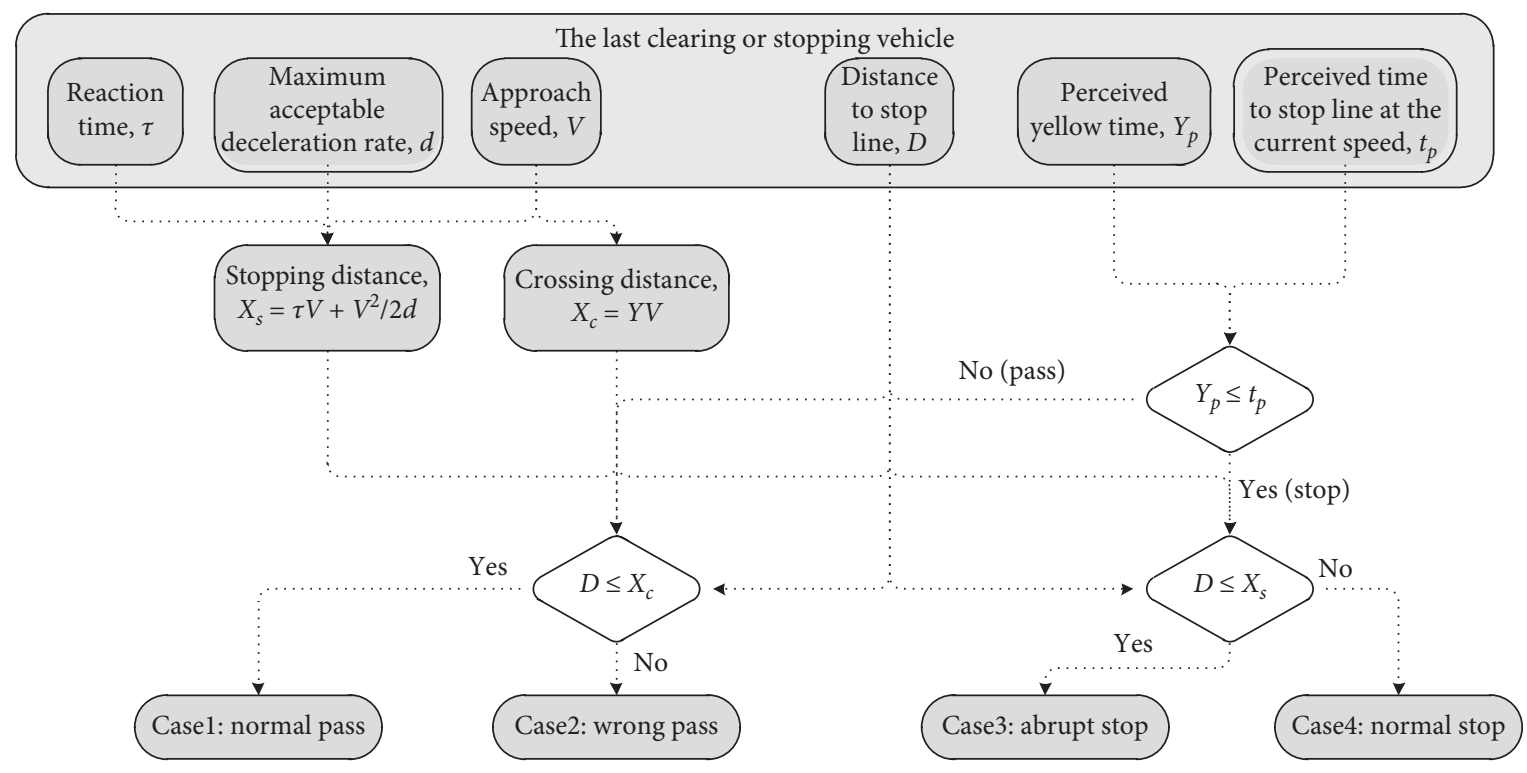

FIGURE 4: Classification of decision-making behavior.

TABLE 2: Comparison of abnormal decision behavior with RLC and without RLC.

\begin{tabular}{lcccccc}
\hline \multirow{2}{*}{ Abnormal decision } & \multicolumn{3}{c}{ Pass decision } & \multicolumn{2}{c}{ Stop decision } \\
& Normal & Wrong & Wrong percentage (\%) & Normal & Abrupt & Abrupt percentage (\%) \\
\hline RLC & 94 & 15 & 13.8 & 93 & 6 & 6.1 \\
Non-RLC & 109 & 33 & 23.2 & 18 & 2 & 10.0 \\
\hline
\end{tabular}

TABLE 3: Stopping probability models for stop-and-go decision.

\begin{tabular}{|c|c|c|c|c|c|c|c|}
\hline Model & Explanatory variable & Coeff. & S.E. & Wald test & $\mathrm{df}$ & Sig. & $\operatorname{Exp}(\mathrm{B})$ \\
\hline \multirow{2}{*}{ Category 1: presence of RLC $=1$} & Potential time (s) & 2.445 & 0.423 & 33.432 & 1 & 0.000 & 11.528 \\
\hline & Constant & -6.620 & 1.190 & 30.946 & 1 & 0.000 & 0.001 \\
\hline \multirow{2}{*}{ Category 2: presence of RLC $=0$} & Potential time (s) & 1.296 & 0.252 & 26.473 & 1 & 0.000 & 3.655 \\
\hline & Constant & -6.709 & 1.093 & 37.713 & 1 & 0.000 & 0.001 \\
\hline \multirow{3}{*}{ All samples } & Potential time (s) & 1.183 & 0.218 & 69.451 & 1 & 0.000 & 6.129 \\
\hline & Presence of RLC & 3.940 & 0.600 & 43.094 & 1 & 0.000 & 51.435 \\
\hline & Constant & -8.876 & 1.003 & 78.282 & 1 & 0.000 & 0.000 \\
\hline
\end{tabular}

Note: $\log$ likelihood (fitting results of model $)=447.892$. Hosmer and Lemeshow test of goodness of fit $(p)=0.329(p=0.329>0.05$ indicates good result). Area under receiver operating curve $=0.979$, which gives validity of model with lower bound of 0.968 and the upper bound of 0.990 ( $95 \%$ confidence interval). Percentage correct $=0.923$. Coeff. $=$ coefficient; S.E. $=$ standard error; $\mathrm{df}=$ degrees of freedom; Sig. $=$ significance. RLC is dummy variable $(0=$ non $-\mathrm{RLC}$, $1=$ RLC).

yellow light is on, and whether an RLC is installed. Table 3 shows each analysis index and index value.

Table 3 shows the -2log likelihood and two pseudodecision coefficients, that, the Cox and Snell $R$ squared and the Nagelkerke $R$ squared of the current model. The smaller the $-2 \log$ likelihood is, the closer it is to 0 and the better the model fit is. The greater the pseudodeterminate coefficient is, the closer it is to 1 and the higher the model precision is. The prediction accuracy of the model shows that correct prediction records account for $91.3 \%$ of all samples, among which the prediction rate of the stopping decisions is $85.7 \%$, and the prediction rate of the passing decisions is $94.0 \%$. The model predictions are highly accurate.
The stopping probability can be expressed as

$$
P_{\text {stop }}=\frac{1}{1+e^{8.876-1.183 * \text { potential time }-3.940 * \operatorname{RLC}(1 \text { or } 0)}},
$$

where $\mathrm{RLC}=1$ represents RLCs installation and 0 is opposite; $P_{\text {stop }}$ is the probability of making a decision to pass.

In the model, the regression coefficient of speed is positive, the regression coefficient of distance is negative, and the regression coefficient of whether there is an RLC installed (i.e., installed RLC $=1$ ) is negative. This shows that the greater the speed of the vehicle, the smaller the distance from the stop line and the higher the probability of making the pass decision when an RLC is not installed. Comparing the absolute values of the coefficient of each influencing factor, we can see that the 
coefficient value of the RLC is far greater than those of speed and distance, which shows that RLCs' installation has a significant impact on the decision-making process of passing, which is consistent with the actual situation.

\subsection{Rear-End Collision Probability Model}

4.2.1. Determination of Key Influencing Parameters. For drivers approaching the stop line when the yellow light is on, they must decide whether to go through or to stop. If the driving speed is high and the vehicle is very close to the stop line, the driver should choose to cross the intersection before red light onset. On the contrary, if the distance is far, the driver should make a decision to brake. Two types of abnormal driving behaviors often occur when drivers make decisions. One is when they are far away from the intersection, they make a decision to pass, which makes them unable to pass the intersection smoothly at a normal speed. In this case, the driver tends to speed up to pass the stop line as fast as possible. This inappropriate action is likely to lead to running a red light and then cause the right-angle collisions with the cross traffic flow. Another situation is when the driver makes a decision to stop when he is close to the intersection, which results in a failure to stop safely in front of the stop line with normal braking. Such decision-making for emergency stopping readily leads to rear-end collisions.

When the trailing car follows the leading car, an incorrect decision by the leading car will lead to rear-end collisions. The necessary condition for rear-end collisions is that the leading car chooses to stop and then the car makes a pass decision. When a yellow light or green light is active, the leading vehicle fails to slow down sufficiently due to an incorrect judgment or perception error, resulting in emergency deceleration. However, if the distance between the following car and the leading car is small and the driving speed exceeds that of the leading car, the possibility of rearend collisions increases.

The stop-and-go decisions made by the driver of the leading vehicle mainly consider the distance to the stop line and the current speed, that is, the potential travel time. Some scholars, such as Mahalel, Zaidel, and Hayward, have clearly pointed out that speed and distance, that is, the potential travel time, have a significant impact on the driver's stop-andgo decision-making. Hence, the potential travel time was used to study the driver's behavior during the period from green to yellow lights [23-25]. In addition, some studies have shown that changes in headway will have an important impact on the decision-making of the following vehicle and thus indirectly affect the possibility of rear-end collisions [16]. Therefore, the headway and the potential travel time are selected as the key influencing factor for rear-end hazard analysis for the process of vehicle decision-making. Under two key traffic parameters, the risk of vehicle rear-end collision under different traffic flow conditions is discussed.

\subsubsection{Probability Analysis of Rear-End Collisions.} Headway refers to the time interval between two consecutive vehicles in a vehicle queue operating in the same lane passing through a certain section. Obviously, headway, as the time difference between two vehicles passing through the parking line, has an impact on rear-end accidents. Therefore, headway will be used as an important variable in the probability analysis of rear-end collisions.

Using the developed decision model and mathematical simulation method, the theoretical probability of rear-end collisions at the RLC intersection under the combination of headway and the potential travel time is obtained, as shown in Figure 5.

Figure 5 shows that the changing trend of the probability of rear-end collisions under different headways is the same, and the maximum occurrence rate is potential travel time value of $2-3 \mathrm{~s}$, which shows that the possibility of inconsistent decision-making between the leading and following vehicles is the largest within $2 s \sim 3 s$ from stopping line. In other intervals, the leading and following vehicles tend to make the same decision.

When the potential travel time takes on a certain fixed value, the smaller the headway is, the greater the incidence of rear-end collisions is. This indicates that the smaller the following distance is, the greater the influence of the leading car is. After being influenced by the driving behavior tendency of the leading car, the following car will take a while to make corresponding adjustments on the driving state. During the reaction time period, the driving speed of the following car may be much faster than that of the leading car, and the time to collision (TTC) decreases sharply, which corresponds to an increased possibility of collision.

\section{Risk Impact Analysis of RLC on Driving Behavior}

5.1. The Influence of RLCs on Drivers' Decision-Making Behavior. The dilemma zone is caused in the process of stop-and-go decision-making, which has always been an important index to evaluate the safety of intersections. There are two types of difficult areas which lead to incorrect decisions [26]. Type I dilemma zone occurs when the intergeen time is insufficient, which leads to the driver being unable to not only achieve a safe stop before the red light turns on but also pass through the intersection safely. Type I dilemma zone is mainly eliminated by the design of the yellow time and all-red time. Type II dilemma zone reflects a region in which it is difficult for drivers to make stop-and-go decisions. Therefore, Type II dilemma zone is also called the "indecision zone." The most typical definition of Type II dilemma zone is the zone between the $10 \%$ probability stop and $90 \%$ probability stop when the yellow light is on. [26-30]. Based on the stop-and-go decision model, Type II dilemma zone of the intersection with or without RLC is established, as shown in Figure 6.

Figure 4 shows that the indecision zone of the RLC intersection is $2 \mathrm{~s}-3.9 \mathrm{~s}$, and the indecision zone of the nonRLC intersection is $3.5 \mathrm{~s}-6.9 \mathrm{~s}$. Compared with the nonRLC intersection, the potential travel times corresponding to $10 \%$ stop probability and $90 \%$ stop probability are $1.5 \mathrm{~s}$ and 3 lower, respectively. This shows that the presence of RLC helps to reduce the difficulty of decision-making by 


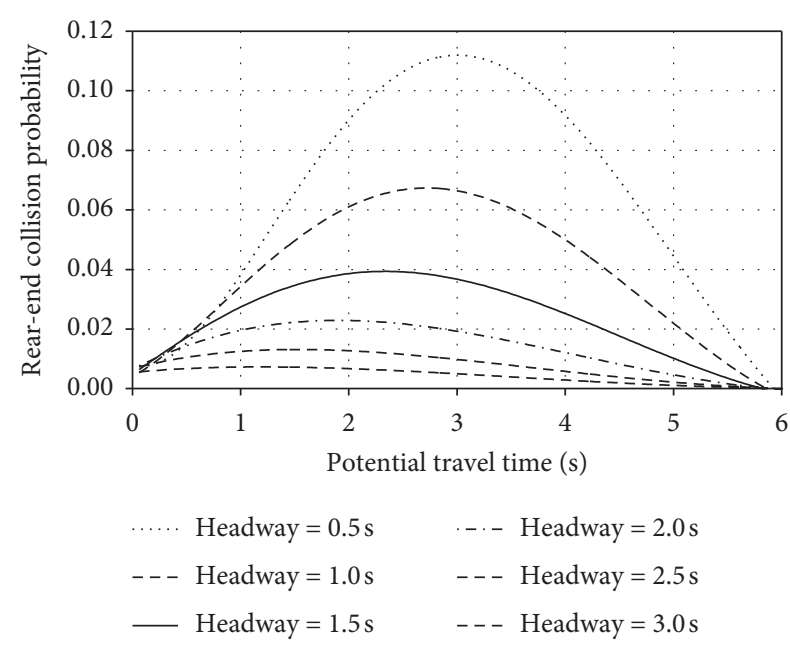

FIgUre 5: Probability of rear-end collisions with different headway.

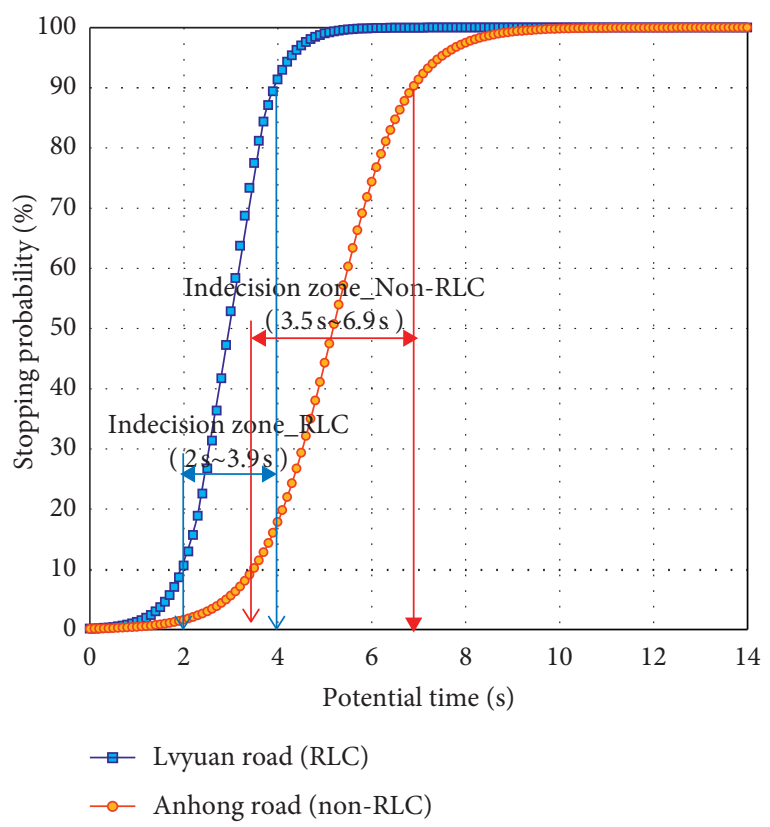

FIGURE 6: Indecision zones of the RLC intersection and the nonRLC intersection.

drivers. This finding is consistent with the results of previous studies [21]; that is, during green or yellow lights, RLCs is very effective in increasing the tendency of drivers to stop.

From the perspective of the time span of the indecision zone, RLCs can help drivers to make decisions more quickly and decisively, which greatly increases the tendency of the leading vehicle to choose to brake. However, the leading vehicle's stop decision is a necessary condition for the occurrence of rear-end accidents. Therefore, although setting RLCs greatly reduces the indecision zone, it brings more possibility to the rear-end accident. The further analysis of the impact of RLCs on rear-end collisions is described in the following content of the paper [31].
5.2. The Influence of RLCs on Risk of Rear-End Collisions. According to the actual observation, the headway of continuous saturated traffic flow is about $2 \mathrm{~s}$. Therefore, taking the headway as $2 \mathrm{~s}$, this paper analyses the impact of different potential travel time on the rear-end collision probability, as shown in Figure 7.

(i) RLCs can effectively reduce the rear-end collision probability in ranges close to or far from the intersection.

(ii) For vehicles with low driving speeds, RLCs will effectively reduce the rear-end collision risk, which indicates that the decision-making tendency of these drivers is greatly affected by RLCs. For vehicles with high driving speeds, RLCs have no obvious influence on the rear-end collision probability because most of these drivers are aggressive, and their decision-making tendency is generally affected by RLCs.

(iii) According to the previous speed analysis, the driving speed of most vehicles is in the range of $30 \mathrm{~km} / \mathrm{h}$ to $70 \mathrm{~km} / \mathrm{h}$; therefore, in the range of potential traffic times of $2 \mathrm{~s} \sim 3 \mathrm{~s}$, RLCs will increase the probability of rear-end collisions for most vehicles.

In this study, estimated time to stop line (ETSL) proposed by Huang et al. was used to analyse the effect of RLCs on the rear-end collision probability with different speeds [16]. Through mathematical simulation, the cut-off speed curves corresponding to the ETSL are obtained when the headway is taken as $2 \mathrm{~s}$. The area above the curve indicates an increase in the rear-end collision probability, as shown in Figure 8.

From Figure 8 , it can be seen that $80 \%$ of the vehicles at non-RLC intersections fall on the top of the curve, while $91 \%$ of the vehicles at RLC intersections fall on the top. On the whole, RLCs can lead to greater probability of rear-end collisions, but the effect of RLCs on the risk of rear-end collision is not obvious in a specific range.

\section{Conclusions}

In this study, aiming at typical problems, such as lag and large errors in accident statistics research methods for analysing the rear-end collision risk with RLCs installed, a simulation analysis method of rear-end collisions based on the probability of collision theory is proposed, and the feasibility of this method is verified using two adjacent, similar intersections in Shanghai. This study mainly considers the driving characteristics, decision-making behavior, and safety status of vehicles with and without RLCs. The study not only analyses these three aspects separately but also discusses the relevance of each analysis conclusion to provide a comprehensive understanding of the impact of RLCs on vehicles at intersections. The following conclusions are drawn in this study:

(1) The method of rear-end collision risk assessment based on mathematical simulation and calculation can determine the rear-end collision probability at 


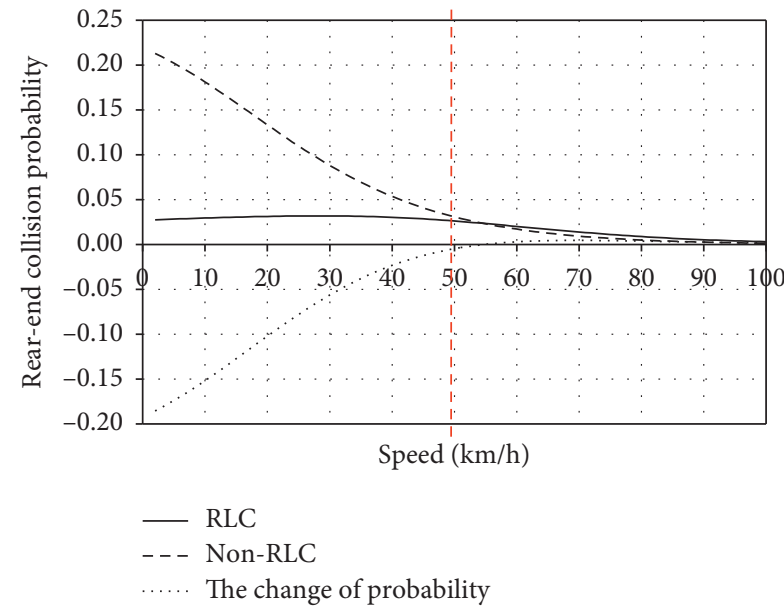

(a)

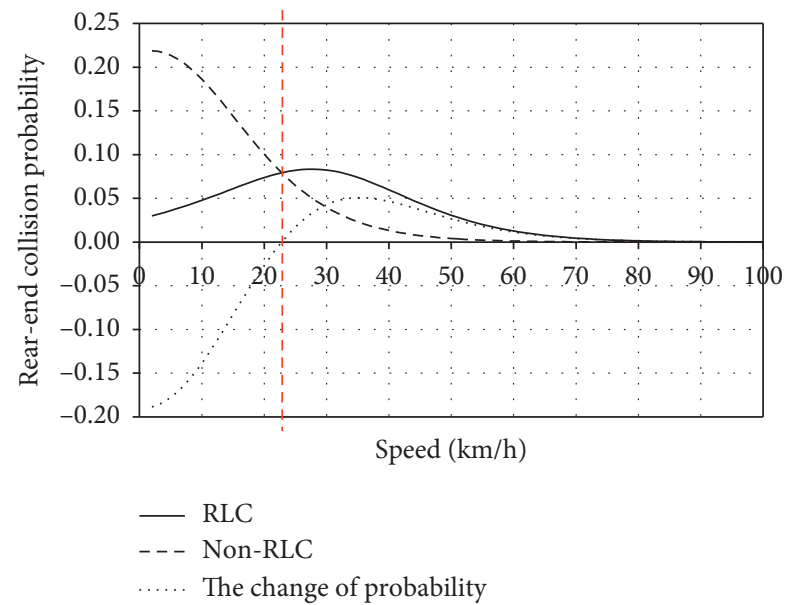

(c)

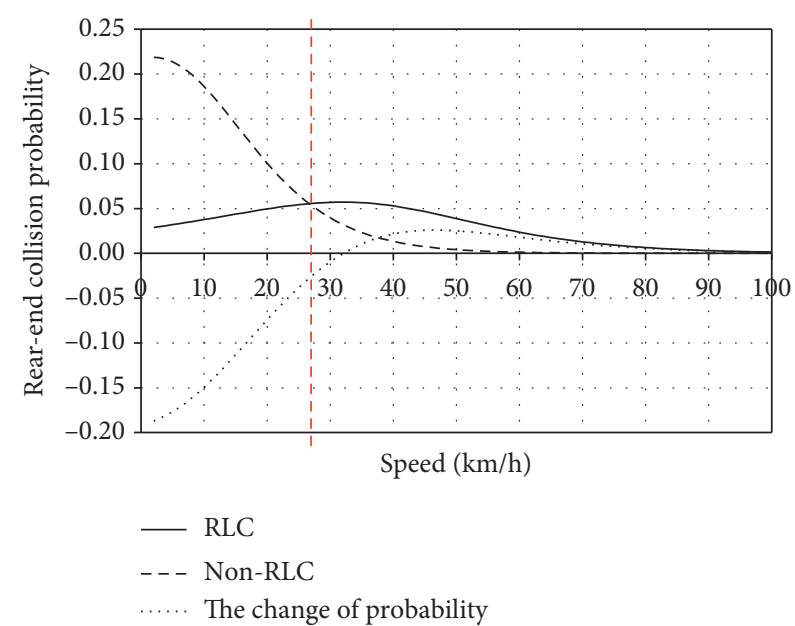

(b)

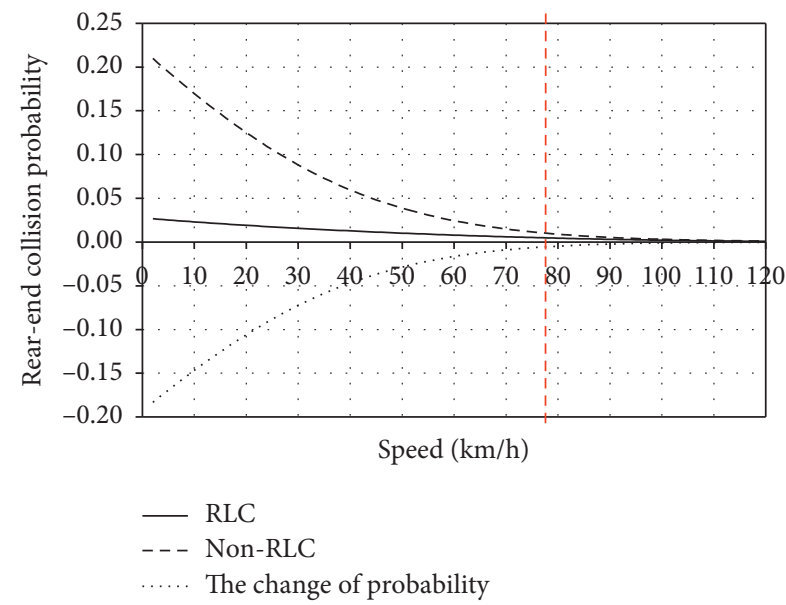

(d)

Figure 7: Probability of rear-end collisions with different potential travel time. Through the analysis of the above probability curve, the following can be found: (a) Potential travel time $=1.0 \mathrm{~s}$. (b) Potential travel time $=2.0 \mathrm{~s}$. (c) Potential travel time $=3.0 \mathrm{~s}$. (d) Potential travel time $=4.0 \mathrm{~s}$.

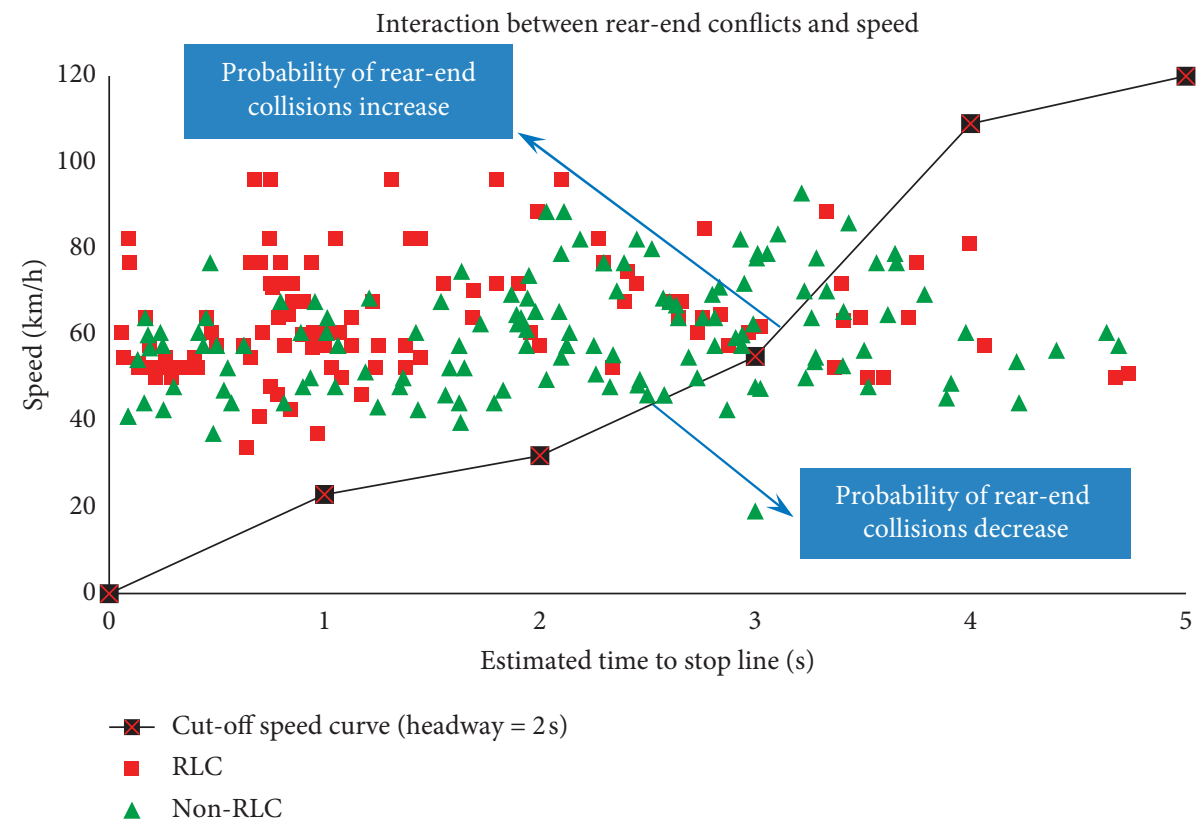

FIgURE 8: The rear-end collision probability distribution with ETSL. 
intersections before and after the installation of RLCs. This method has wide applicability.

(2) RLCs have an important impact on the driver's speed. They can cause the vehicle speed at the intersection to increase and cause more abnormal driving behaviors, which increase the difficulty of driver's stop-and-go decision-making.

(3) RLCs are helpful for drivers to make the stop-and-go decision, reduce the difficulty of decision, and increase the tendency of stopping and the complexity and possibility of rear-end collision.

(4) Unlike previous studies, we find that RLCs do not always lead to an increase of rear-end collisions. For the vehicle close to or far from the intersection at the decision-making time, the RLC will significantly reduce the rear-end collision probability; however, for the vehicle at the potential travel time of $2 \mathrm{~s} \sim 3 \mathrm{~s}$, RLCs will cause an increase of rear-end collisions. RLCs have minimal effects on aggressive drivers who drive at high speed at decision-making time.

(5) The probability distribution of rear-end collisions under different traffic flow states shows that strict speed limit management can lower the rear-end collision risk caused by RLCs.

\section{Data Availability}

The data used to support the findings of this study are available from supplemental files.

\section{Conflicts of Interest}

The authors declare that there are no conflicts of interest regarding the publication of this paper.

\section{Acknowledgments}

This study was supported by Public Technology Research Program of Zhejiang Province (no. LGF20F030004), Natural Science Foundation of Ningbo Municipality (no 2019A610044), and Opening Foundation of the State Key Laboratory for Management and Control of Complex Systems (no. 20190102). The authors are grateful to Professor Hideki Nakamura at Nagoya University for providing the image-processing software for data reduction in this study. Thanks are also due to Dr. Jibiao Zhou at Ningbo University of Technology for data collection and reduction as well as the construction of stop-and-go decision-making model.

\section{Supplementary Materials}

This section includes two Excel tables. Table S1: data for building indecision zone models of the RLC intersection and the non-RLC intersection (Figure 6 in the article). Table S2: all simulation data of rear-end probability calculation. Using these data, we can build the probability of rear-end collision models (Figures 5, 7, and 8 in the article). (Supplementary Materials)

\section{References}

[1] H. W. McGee and K. A. Eccles, "Impact of red light camera enforcement on crash experience," ITE Journal, vol. 73, no. 3, pp. 44-48, 2003.

[2] Safety Evaluation of Red-Light Cameras, RRPORT FHWAHRT-05-048, Federal Highway Administration, Washington, DC, USA, 2005.

[3] F. Chen, M. Song, and X. Ma, "Investigation on the injury severity of drivers in rear-end collisions between cars using a random parameters bivariate ordered probit model," International Journal of Environmental Research and Public Health, vol. 16, no. 14, Article ID 2632, 2019.

[4] F. Chen and S. Chen, "Injury severities of truck drivers in single- and multi-vehicle accidents on rural highways," Accident Analysis \& Prevention, vol. 43, no. 5, pp. 1677-1688, 2011.

[5] C. Ma and Y. Dong, "Public transit network planning in small cites considering safety and convenience," Advances in Mechanical Engineering, vol. 12, no. 1, pp. 1-12, 2020.

[6] C. Ma, Y. Dong, J. Zhou, Z. Feng, and Y. Quan, "Risk riding behaviors of urban E-bikes: a literature review," International Journal of Environmental Research and Public Health, vol. 16, no. 13, Article ID 2308, 2019.

[7] H. C. Chin, "Effect of automatic red-light cameras on red running," Traffic Engineering and Control, vol. 30, no. 4, pp. 175-179, 1989.

[8] C. H. Ng, Y. D. Wong, and K. M. Lum, "The impact of red light surveillance cameras on road safety in Singapore," Road and Transport Research, vol. 6, no. 2, pp. 72-81, 1997.

[9] G. E. Maisey, "The effects of mechanical surveillance device on urban signalized intersection accidents," Research and Statistics Report no. 17, Road Traffic Authority, Perth, Australia, 1981.

[10] D. R. South, W. A. Harrison, I. Portans, and M. King, Evaluation of the Red Light Camera Program and the Owner Onus Legislation, Victoria Transport: Road Traffic Authority, Hawthorn, Victoria, Australia, 1998.

[11] W. Hillier, J. Ronczka, and F. Schnerring, An Evaluation of Red Light Cameras in Sydney, Road Safety Bureau, Roads and Traffic Authority, New South Wales, Australia, Research Note RN 1/93, 1993.

[12] D. A. Andreassen, Long Term Study of Red Light Cameras and Accidents, Research Report ARR No. 261, ARRB Transport Research Ltd., Victoria, Australia, 1995.

[13] R. A. Retting and S. Y. Kyrychenko, Crash Reductions Associated with Red Light Camera Enforcement in Oxnard, California, Insurance Institute for Highway Safety, Arlington, VA, USA, 2001.

[14] P. B. Farradyne, City of San Diego Photo Enforcement System Review Final Report, City of San Diego Police Department, Los Angeles, CA, USA, 2002.

[15] U.S. House of Representatives, The Red Light Running Crisis, Is it Intentional, Office of the Majority Leader, U.S. House of Representatives, Washington, DC, USA, 2001.

[16] H. L. Huang, H. C. Chin, and A. H. H. Heng, "“Effect of red light cameras on accident risk at intersections", transportation research record," Journal of the Transportation Research Board, vol. 1969, pp. 18-26, 2006.

[17] Q. Zeng, W. Gu, X. Zhang, H. Wen, J. Lee, and W. Hao, "Analyzing freeway crash severity using a Bayesian spatial generalized ordered logit model with conditional autoregressive priors," Accident Analysis \& Prevention, vol. 127, pp. 87-95, 2019. 
[18] Q. Zeng, H. Wen, H. Huang, and M. Abdel-Aty, "A Bayesian spatial random parameters Tobit model for analyzing crash rates on roadway segments," Accident Analysis \& Prevention, vol. 100, pp. 37-43, 2017.

[19] J. Sun, T. Li, F. Li, and F. Chen, "Analysis of safety factors for urban expressways considering the effect of congestion in Shanghai, China," Accident Analysis \& Prevention, vol. 95, pp. 503-511, 2016.

[20] B. Dong, X. Ma, F. Chen, and S. Chen, "Investigating the differences of single- and multi-vehicle accident probability using mixed logit model," Journal of Advanced Transportation, Article ID 2702360, 9 pages, 2018.

[21] K. M. Lum and Y. D. Wong, "A before-and-after study of driver stopping propensity at red light camera intersections," Accident Analysis and Prevention, vol. 35, no. 2, pp. 111-120, 2003.

[22] J. Sun, X. S. Dong, and Y. Ni, "Comparative study of impacts of red light cameras in China," Transportation Research Record: Journal of the Transportation Research Board, vol. 2317, no. 1, pp. 68-75, 2012.

[23] H. Köll, M. Bader, and K. W. Axhausen, "Driver behavior during flashing green before yellow: a comparative study," Accident Analysis and Prevention, vol. 36, no. 2, pp. 273-280, 2004.

[24] D. Mahalel and D. M. Zaidel, "Safety evaluation of a flashinggreen light in a traffic signal," Traffic Engineering and Control, vol. 26, no. 2, pp. 79-81, 1985.

[25] J. C. Hayward, "Near-miss determination through use of a scale of danger," Highway Research Record, vol. 384, pp. 2434, 1972.

[26] T. J. Gates, D. A. Noyce, and L. Laracuente, "Analysis of dilemma zone driver behavior at signalized intersections," in Proceedings of the Transportation Research Board 85th Annual Meeting, Transportation Research Board, Washington, DC, USA, 2006.

[27] B. James and K. Zimmerman, Red-Light-Running Handbook: An Engineer's Guide To Reducing Red-Light-Related Crashes, Texas Department of Transportation and the Federal Highway Administration, Austin, TX, USA, 2004.

[28] B. E. Porter and T. D. Berry, "A nationwide survey of selfreported red light running: measuring prevalence, predictors, and perceived consequences," Accident Analysis \& Prevention, vol. 33, no. 6, pp. 735-741, 2001.

[29] C. V.Zegeer, Effectiveness of Green-Extension Systems at HighSpeed Intersections, Kentucky Transportation Center at the University of Kentucky, Lexington, KY, USA, 1977.

[30] A. D. May, Clearance Interval at Flashing Systems, Highway Research Board, National Research Council, Washington, DC, USA, Highway Research Record 221, 1968.

[31] S. Dong, J. Zhou, and S. Zhang, "Determining E-bike drivers' decision-making mechanisms during signal change interval using the hidden markov driving model," Journal of Advanced Transportation, vol. 2019, Article ID 7341097, 10 pages, 2019. 